\title{
Obstacle Avoidance of a Redundant Robot Using Virtual Force Field and Null Space Projection
}

\author{
Yiming Jiang ${ }^{1}$, Chenguang Yang ${ }^{1,2 *}$, Zhaojie $\mathrm{Ju}^{3}$, and Jinguo $\mathrm{Liu}^{4}$ \\ ${ }^{1}$ College of Automation Science and Engineering, South China University of Technology, \\ Guangzhou 510640, China \\ 2 Bristol Robotics Laboratory, University of the West of England, Bristol BS16 1QY, UK \\ ${ }^{3}$ School of Computing, University of Portsmouth, Portsmouth PO1 3HE, UK \\ ${ }^{4}$ Institutes for Robotics and Intelligent Manufacturing, Chinese Academy of Sciences, \\ Shenyang 110016, China \\ *Corresponding author: cyang@ieee.org
}

\begin{abstract}
This paper presents a novel algorithm for redundant robot control when obstacles are approaching to the robot. The proposed controller is constructed by a multi-hierarchy control framework where a main task controller is designed to track a given Cartesian space trajectory and an extra impedance controller is developed in the null space to guarantee compliant joint motion. A virtual force field is designed and applied on the robot body to achieve the goal of the avoidance of the obstacle. Simulation studies illustrated the proposed controller is not only to guarantee the task space control, but also able to avoid the obstacle by joint movements.
\end{abstract}

\section{Introduction}

In recent years, the potential of physical interactions in human robot co-existent scenarios has elicited large interests in industry and academia and leads to the recent growth of research in physical human-robot interactions (pHRI) [1-6]. Redundant robots are well known for their abundant joint actuators which are useful to enhance the dexterous of the robot motion. This is based on the fact that the redundant robot has more degree of freedom (DOF) than required to complete a given task. However, the increased DOFs generate complexity of the controller design, which is regarded as a challenge problem as the inverse kinematic solution of the robot is not unique. In order to address the control problem of the redundant robot system, many works have been presented in the past researches.

To solve kinematic control of a redundant manipulator, the pseudo-inverse of the Jacobian was used to calculate the minimum-morn joint velocity for the desired endeffector velocity [7]. But repetitive joint motion is not guaranteed from the repetitive end-effector motion. In [8], a dual neural network is proposed to solve the inverse kinematics problem of redundant robots. In this kinematics control scheme, the kinematic equation is described as a bi-criteria of the infinity and Euclidean norms, in which the constraints of the robot joint position and velocity are incorporated. In [9], a trajectory planning method is proposed for redundant robots by combining a closed-loop pseudoinverse and the genetic algorithm, and in this way the trajectory planning problem 


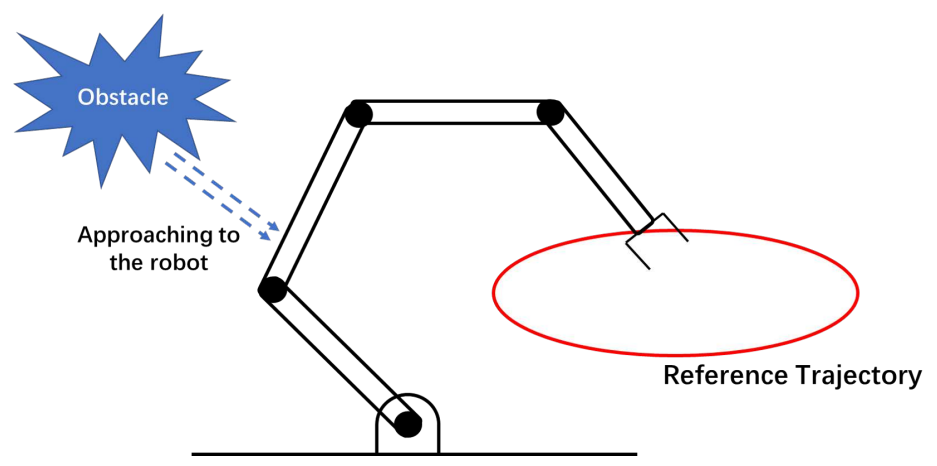

Fig. 1. An overview of the 4 DOF planar robot

can be reformulated as an optimization problem. In this work, the inverse solution of the kinematics is derived such that proper joint angles can be generated.

For the design of kinematic control of redundant robots, the null space of Jacobian matrix is extensively investigated to maintain manipulability or avoid obstacles while finish the required end-effector tasks. In [10], a dynamic feedback control law was presented by providing redundancy resolution with null-space joint velocity tracking. In [11], a velocity control algorithm was proposed in an extended operational space to guarantee stable operations based on a minimal null-space projection. In these studies, the robot dynamics is decomposed into two parts, the task space dynamics and the null space dynamics. In this way specific self motion of the joint can be designed by optimizing a certain function. But how to select a proper optimize function is still an open problem.

The obstacle avoidance is an important issue and therefore has been widely investigated during the past decades. In [12], by dividing the required task into subtasks, a task-priority based redundancy control is proposed, in which the low priority subtask can be performed on joint motion so that the obstacle avoidance can be accomplished in the low priority task. In [13], the optimization strategy is generated for a redundant manipulator subject to the constraints of a mobile object. In [14], an extended Jacobian method was proposed for the obstacle avoidance by using an optimization of a criterion function. However, algorithm singularities may arise due to the restriction of the motion. It should be emphasized that the design of the kinematic control could be much difficult if there are obstacles existed in the workspace of the robot. Hence, it is necessary to design an appropriate null motion to enhance the control performance of obstacle avoidance.

Note that artificial potential field method has been widely applied to deal with the obstacle avoidance problem for mobile robot or manipulator. The basic idea is to design the control of robot movement due to a virtual potential field where the target point generates "gravitational force" to the mobile robot, and the obstacle generates a "repulsive force" to the mobile robot [15]. A potential field representation is proposed to deal with the path planning problem of a three dimensional mover [16]. 


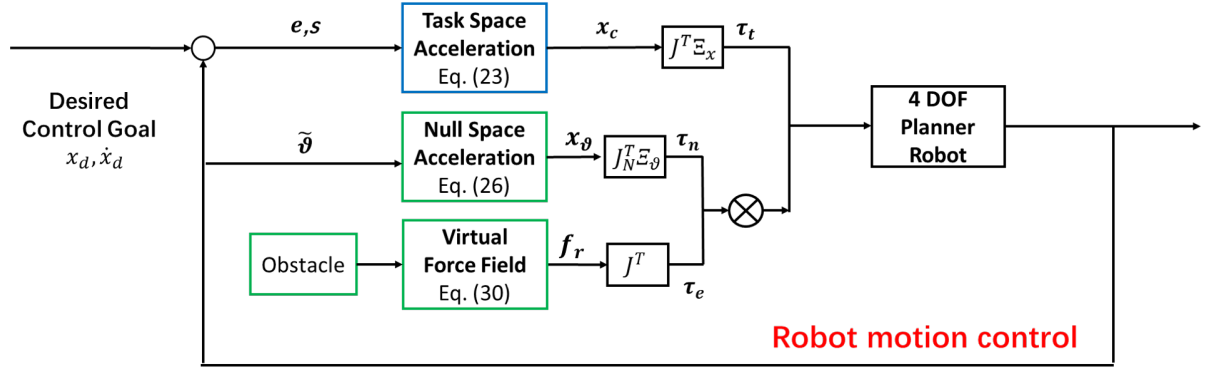

Fig. 2. Control block of our proposed motion controller

Inspired by the works mentioned above, in this paper, we introduce a concept of virtual force field where the obstacle can generates a repulsive force field and apply on the robot. Additionally, an impedance control law is integrated into the null space control, such that self motion of robot can been performed according to the resulted virtual force applied on robot.

\section{System Description and Control objective}

The system we studied in this paper is a redundant robot as shown in Fig.1, where the robot could be a 4-DOF planer redundant robot while an obstacle is approaching to the robot during the movement. The control objective of the robot is to track a desired trajectory in the Cartesian space while to avoid the possible collision to the obstacle.

\subsection{Kinematics of a Redundant}

Consider a redundant robot which has $n$ revolute joints and operates in an $m$ dimensional Cartesian space with $m<n$, the differential kinematic equation can be described as follows,

$$
\dot{x}=J(q) \dot{q}
$$

where $\dot{x}$ is the Cartesian space velocity, $q$ and $\dot{q}$ are the position and velocity of the robot joints, respectively, and $J(q)$ is the Jacobian matrix from the robot end-effector to the joints.

The inverse kinematics of a redundant robot (1) can be generally formulated as

$$
\dot{q}=J^{\#}(q) \dot{x}+N \zeta
$$

where $J^{\#}(q)$ satisfies $J=J J^{\#} J$, denoting the generalized inverse of $J(q), N=$ $I-J^{\#} J$ is the null space of the Jacobian Matrix $J(q)$, while $\zeta$ an arbitrarily selected vector. 


\subsection{Robot Dynamics}

The robot dynamic system is formulated by using the Lagrange formulation as below,

$$
M(q) \ddot{q}+C(q, \dot{q}) \dot{q}+G(q)=\tau^{\prime}+J^{T}(q) f(t)
$$

where $M(q), C(q, \dot{q})$ and $G(q)$ are the inertia/mass matrix, Coriolis/centrifugal matrix and gravity vector, respectively, $f(t)$ is the external force applied on the robot endeffector by the human arm, and $\tau^{\prime}$ is the actuated torque of the joint.

To facilitate the formulation, let us introduce the control signal as follows

$$
\tau^{\prime}=C(q, \dot{q}) \dot{q}+G(q)+\tau
$$

Then the system dynamics can be rewritten as

$$
M(q) \ddot{q}=\tau+J^{T}(q) f(t)
$$

\subsection{Extended Jacobian Formulation}

For a redundant robot described in (2), there are $m$ DOFs in task space and $n$ DOFs in joint space with $n>m$. Thus we have $r=n-m$ redundant DOFs. Then we can rewrite the velocity in null space as

$$
\dot{q}_{n}=J_{N}(q) \vartheta=N \zeta
$$

with $\dot{q}_{n}$ being the redundancy solution, $J_{N}(q)$ is a selected $n \times r$ full column rank matrix to satisfy $J(q) J_{N}(q)=\mathbf{0}$, and $\vartheta$ is a vector of the null space velocity and defined as

$$
\vartheta=J_{N}^{\#}(q) \dot{q}_{n}
$$

with $J_{N}^{\#}$ being the generalized inverse of $J_{N}(q)$ and defined as

$$
J_{N}^{\#}=\left(J_{N}^{T} W_{J_{N}} J_{N}\right)^{-1} J_{N}^{T} J_{N}
$$

where $W_{J_{N}}$ is a weight matrix. In this way, $\vartheta$ can be designed to realized the null space motion without affecting the motion in Cartesian space.

By combining (1) and (7), an extended velocity vector $\dot{x}_{e}$ is constructed as

$$
\dot{x}_{e}=\left[\begin{array}{c}
\dot{x} \\
\vartheta
\end{array}\right]=\left[\begin{array}{c}
J(q) \\
J_{N}^{\#}(q)
\end{array}\right] \dot{q}
$$

Let $J_{e}=\left[\begin{array}{c}J(q) \\ J_{N}^{\#}(q)\end{array}\right]$, and taking the time derivative of (9), we have

$$
\ddot{x}_{e}=\left[\begin{array}{l}
\ddot{x} \\
\dot{\vartheta}
\end{array}\right]=\left[\begin{array}{l}
J(q) \ddot{q}+\dot{J}(q) \dot{q} \\
J_{N}^{\#}(q) \ddot{q}+\dot{J}_{N}^{\#} \dot{q}
\end{array}\right]
$$

According to the definition of $J_{e}$, (10) can be rewritten as

$$
\ddot{x}_{e}=J_{e} \ddot{q}+\dot{J}_{e} \dot{q}
$$


As the properties $J_{e}^{\#}(q) J_{e}=I$ and $J_{e} J_{e}^{\#}(q) J_{e}=J_{e}$ holds for $J_{e}^{\#}(q)$, we can define the generalized inverse of $J_{e}(q)$ as $J_{e}^{\#}(q)=\left[J^{\#}(q) J_{N}(q)\right]$. Then premultiplying $J_{e}^{\#}$ on both sides of (11), we can obtain

$$
\ddot{q}=J_{e}^{\#}\left(\ddot{x}_{e}-\dot{J}_{e} \dot{q}\right)
$$

From the above theorem, the convergence of the interaction force is achieved.

\section{Control Design for the Redundant Robot}

\subsection{Decoupled System Dynamics}

Substituting (12) into (5), and considering $\dot{q}=J^{\#} \dot{x}+J_{N}(q) \vartheta$, then the system dynamics can be rewritten as

$$
J_{e}^{\# T} \tau=\Xi_{e}(q) \ddot{x}_{e}+\eta_{e} \dot{x}_{e}+J_{e}^{\# T} J^{T} f(t)
$$

where

$$
\begin{aligned}
& \Xi_{e}=J_{e}^{\# T} M J_{e}^{\#}=\left[\begin{array}{cc}
J^{\# T} M J^{\#} & J^{\# T} M J_{N} \\
J_{N}^{T} M J^{\#} & J_{N}^{T} M J_{N}
\end{array}\right] \\
& \eta_{e}=J_{e}^{\# T} \dot{J}_{e} J_{e}^{\#}
\end{aligned}
$$

Note that the matrix $\Xi_{e}$ in (14) is not diagonal. The off-diagonal terms lead to the coupled dynamics between the task space and null space. In order to control the task space motion while achieve specified null space motion, these coupled terms should be appropriately cancelled. Motivated by the work in [17], we introduce an inertiaweighted generalized inverse by choosing $W_{J}=W_{J_{N}}=M$, such that

$$
\left\{\begin{array}{l}
J^{\#}=M^{-1} J^{T}\left(J M^{-1} J^{T}\right)^{-1} \\
J_{N}^{\#}=\left(J_{N}^{T} M J_{N}\right)^{-1} J_{N}^{T} M
\end{array}\right.
$$

Since we have $J(q) J_{N}(q)=0$, the equality $J^{\# T} M J_{N}=J_{N} M J^{\#}=0$ holds. Then, $\Xi_{e}$ becomes

$$
\Xi_{e}=\left[\begin{array}{cc}
J^{\# T} M J^{\#} & 0 \\
0 & J_{N}^{T} M J_{N}
\end{array}\right]
$$

From (16) we can see that $\Xi_{e}$ is a diagonal matrix. Hence, we can design the controller for the two subsystems respectively.

$$
\left\{\begin{array}{c}
J^{\# T} \tau_{x}=J^{\# T} M J^{\#} \ddot{x}+\eta_{x} \dot{x}+J^{\# T} J^{T}(q) f(t) \\
J_{N}^{T} \tau_{n}=J_{N}^{T} M J_{N} \dot{\vartheta}+\eta_{\vartheta} \vartheta+J_{N}^{T} \tau_{e}
\end{array}\right.
$$

where $\eta_{x}=\left[\begin{array}{l}J^{\# T} \dot{J}(q) J^{\#}(q) \\ J^{\# T} \dot{J}_{N}^{\#}(q) J_{N}(q)\end{array}\right], \eta_{\vartheta}=\left[\begin{array}{l}J_{N}^{T} \dot{J}_{N}^{\#}(q) J^{\#}(q) \\ J_{N}^{T} \dot{J}(q) J_{N}(q)\end{array}\right]$, and $\tau_{e}$ is external torque applied on the robot. Note that the property $J(q) J_{N}(q)=\mathbf{0}$ holds, therefore the two 
subsystems in the above formulation are orthogonal to each other. Also, $\tau_{e}$ is in the null space of the main task. We rewritten (17) compactly as

$$
\left\{\begin{array}{l}
J^{\# T} \tau_{x}=\Xi_{x} \ddot{x}+\eta_{x} \dot{x}+f(t) \\
J_{N}^{T} \tau_{n}=\Xi_{\vartheta} \dot{\vartheta}+\eta_{\vartheta} \vartheta+J_{N}^{T} \tau_{e}
\end{array}\right.
$$

where $\Xi_{x}=J^{\# T} M J^{\#}, \Xi_{\vartheta}=J_{N}^{T} M J_{N}$.

\subsection{Design of the Controller}

Error signal Remind that the objective of the robot inter-loop controller is to track the given trajectory, the tracking error is defined as

$$
\begin{aligned}
& e=x-x_{r} \\
& s=\dot{x}-\dot{x}_{s}
\end{aligned}
$$

where $\dot{x}_{s}=\dot{x}_{r}+K_{s} e$ with $K_{s}$ being a positive definite matrix. Note $x$ and $x_{r}$ are uniformly continuous and bounded, thus the convergence of the signal $s$ could lead to the convergence of $\dot{e}$ and $e$.

Task space control To track a given trajectory $x_{r}$, the acceleration control command $x_{c}$ is designed as follows,

$$
\ddot{x}_{c}=\ddot{x}_{s}-\Xi_{x}^{-1}\left(K_{p}+\eta_{x}\right) s+\Xi_{x}^{-1} \eta_{x} \dot{x}+\Xi_{x}^{-1} J^{T} \hat{f}(t)
$$

where $K_{p}$ is a positive definite matrix. Combining (21) and (17), we can design the control law in task space as,

$$
\tau_{x d}=J^{T} \Xi_{x}\left(\ddot{x}_{s}-\Xi_{x}^{-1}\left(K_{p}+\eta_{x}\right) s+\Xi_{x}^{-1} \eta_{x} \dot{x}\right)+J^{T} \hat{f}(t)
$$

where $\hat{f}(t)$ is the measurement of $f(t)$. Then, substituting (22) into the first equation of (17), the error dynamics of the system can be obtained as,

$$
\Xi_{x} \dot{s}=-\left(K_{p}+\eta_{x}\right) s+J^{T}(q) \tilde{f}(t)
$$

with $\tilde{f}(t)=f(t)-\hat{f}(t)$.

Null space control In order to control the motion of the null space, we select the null space control law as

$$
\dot{\vartheta}_{c}=\dot{\vartheta}_{d}+\Xi_{\vartheta}^{-1}\left(\left(K_{\vartheta}+\eta_{\vartheta} \tilde{\vartheta}\right)+\eta_{\vartheta} \vartheta+J_{N}^{T} K_{v} \tilde{q}\right)
$$

where $\vartheta_{d}$ is the desired null space command, $\tilde{\vartheta}=\vartheta-\vartheta_{d}, K_{\vartheta}$ and $K_{v}$ are positivedefinite symmetric matrices selected by the designer, respectively. Thus, the null space control law can be described as follows,

$$
\tau_{n d}=J_{N}^{\# T}\left(\Xi_{\vartheta} \dot{\vartheta}_{d}+\left(K_{\vartheta}+\eta_{\vartheta}\right) \vartheta+\eta_{\vartheta} \vartheta+J_{N}^{T} K_{v} \tilde{q}\right)
$$

Substitute (24) into (17), we can obtain the closed-loop dynamics of the null motion as below,

$$
\Xi_{\vartheta} \dot{\tilde{\vartheta}}+\left(K_{\vartheta}+\eta_{\vartheta}\right) \tilde{\vartheta}+J_{N}^{T} K_{v} \tilde{q}=J_{N}^{T} \tau_{e}
$$

with $\tau_{e}$ being the external torque applied on the robot. 


\subsection{Virtual Force Field}

In this part, a virtual force field is designed to achieve the obstacle avoidance through null motion. To drive the robot body move away from the obstacle, path planning methods such as configuration space, probabilistic road maps [18], randomly exploring randomized trees [19] and potential field method have been widely investigated. Herein, we employ a force vector field on obstacle to generate external repulsive forces on robot manipulator. Then self motion of the robot can be then performed by the impedance property of the null motion control.

For each link of the robot, a potential energy $U_{i r}$ is designed as,

$$
U_{i r}\left(\boldsymbol{p}_{\boldsymbol{i}}\right)= \begin{cases}0 & \eta_{i}>\eta_{0 i} \\ \frac{1}{2} \gamma_{i}\left(\frac{1}{\eta_{i}\left(\boldsymbol{p}_{\boldsymbol{i}}\right)}-\frac{1}{\eta_{0 i}}\right)^{2} & \eta_{\min }<\eta_{i}\left(\boldsymbol{p}_{\boldsymbol{i}}\right) \leq \eta_{0 i} \\ \nu_{\max } & \eta_{i}\left(\boldsymbol{p}_{\boldsymbol{i}}\right) \leq \eta_{\min }\end{cases}
$$

where $\gamma_{i}$ is the scaling factor represented the intensity of the repulsive force, $\boldsymbol{p}_{\boldsymbol{i}}$ is a control point on the $i$ th robot link, $\eta_{i}\left(\boldsymbol{p}_{\boldsymbol{i}}\right)$ denotes the distance between the $i$ th link and the obstacle, $\eta_{0 i}$ is the effective range of the force field, $\nu_{\max }$ denotes the maximum repulsive force and $\eta_{\min }$ is the threshold to avoid very large force.

Then we can calculate the resulted repulsive force $f_{i r}$ applied on $i$ th robot link as

$$
f_{i r}=-\nabla U_{i r}= \begin{cases}0 & \eta_{i}>\eta_{0 i} \\ \gamma_{i}\left(\frac{1}{\eta_{i}\left(\boldsymbol{p}_{\boldsymbol{i}}\right)}-\frac{1}{\eta_{0 i}}\right) \nabla \eta_{i}\left(\boldsymbol{p}_{\boldsymbol{i}}\right) & \eta_{\min }<\eta_{i} \leq \eta_{0 i} \\ f_{\max } & \eta_{i} \leq \eta_{\min }\end{cases}
$$

where $f_{\text {max }}$ denote the maximum repulsive force, and $\nabla \eta_{i}=\frac{\partial \eta_{i}}{\partial p}$ is the gradient vector. Then, the joint torque can be obtained through the Jacobian matrix of the $i$ th control point as,

$$
\tau_{e}=-\sum_{i=1}^{n} J_{i}^{T} f_{i r}
$$

where $J_{i}$ is Jacobian matrix with respect to the control point $\boldsymbol{p}_{i}$.

Noted that the impedance model regulates the system dynamics concerning the interaction force and motion error. Here, we employ the impedance controller to regulate the null space motion of the robot under the virtual repulsive force produced from the obstacle.

\section{Simulation Studies}

In this section, a group of simulation studies is performed to verify the effectiveness of our proposed controller. The robot is a 4-DOF planar robot as shown in Fig. 1. Each link is weighted with $1 \mathrm{~kg}$ located at the centre of the link. The length of each link is chosen the same as $1 \mathrm{~m}$. The robot has 4-DOF while the required task is 2 DOF tracking.

In the simulation study, totally 2 cases are performed. In this first simulation case, the robot tracks a desired point in the workspace. While a planer circle disk is slowly approaching to the robot. The virtual force filed is generated in term the position of the disk center. 

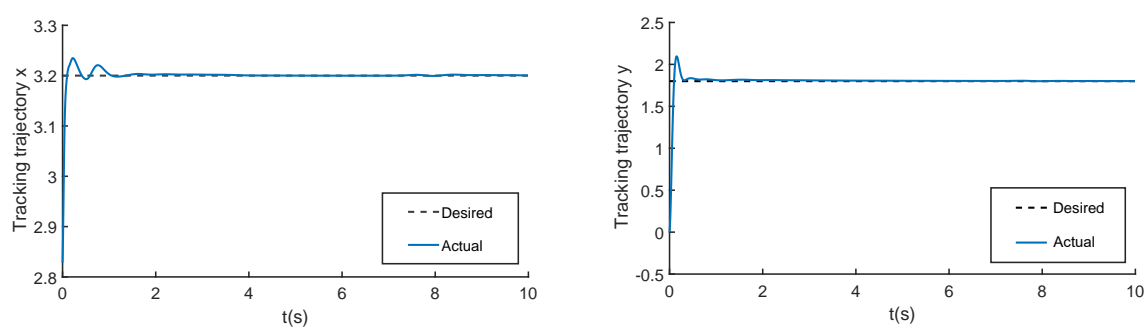

Fig. 3. Tracking trajectory of the proposed con- Fig. 4. Tracking trajectory of the proposed controller $x$ direction troller $y$ direction
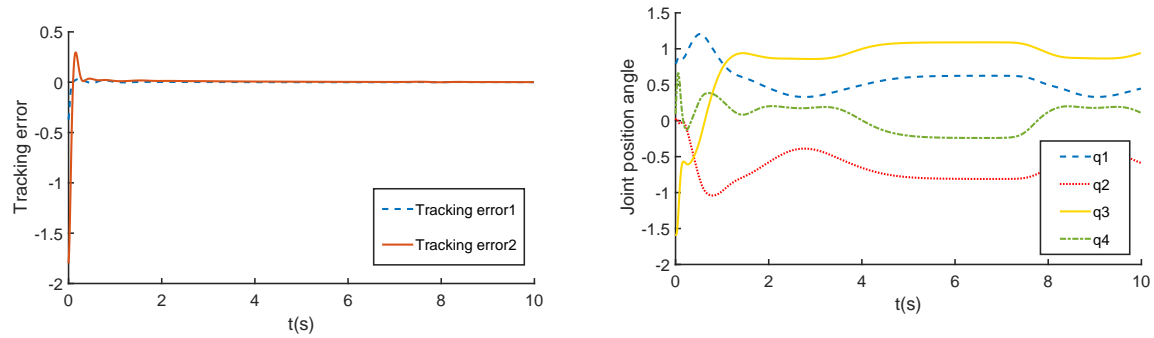

Fig. 6. The joint angles of the robot during track-

Fig. 5. Tracking error of the proposed controller $\begin{array}{r}\text { Fig. } \\ \text { ing }\end{array}$

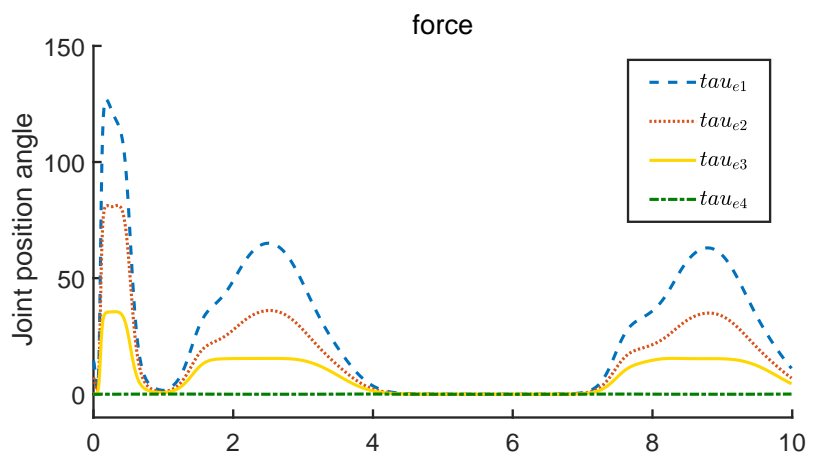

$t(s)$

Fig. 7. The applied repulsive force $\tau_{e}$ on each joint 
The robot initial configuration is chosen to be $q=[p i / 4,0,-p i / 2,0]^{T}$, and the initial position of the obstacle centre is chosen to be $[1.8,2.1]^{T}$. The robot is controlled to track a set position at $x_{d}=[3.2,1.8]^{T}$. The obstacle is movable and follows a sinusoidal trajectory $y(t)=2.1-\sin (t-1)$ in $y$ direction.

The simulation results are performed in Figs.3 - 7. Figs.3-4 depict the tracking performance of the given point tacking task. Also in Fig. 5, we can see that the robot end-effector well follows the reference trajectory, and the tracking errors converge to zero. The applied virtual repulsive force from the obstacle on each joint is performed in Fig. 7. We can see that the applied force increased when the obstacle approaches the robot and reduced when obstacle away from the robot.

The trajectory of all joints angle is depicted shown in Fig.6. We can see the movement of all joint angles according to the change of force field. Also, we can see that in the same period, the end-effector is able to hold on at the fix position. This is because that the effect of virtual forces mainly adds on the null motion of the robot, and the resulted self motion of joints doesn't disturb the end-effector motion. Thus the control goal of the avoidance of a mobile robot is achieved.

In this second simulation case, the robot track a desired trajectory while an obstacle is set on the path of robot movement. The initial configuration of the robot is chosen as the same as the first simulation case, and the initial position of the centre of the obstacle is chosen to be $[1.6,2.1]^{T}$. The robot is controlled to track a trajectory as $x_{d}=\left[2.5,0.5-0.04 t^{3}+0.06 t^{2}\right]$.

The simulation results can be illustrated in Figs.8 - 12. Figs.8-9 show the trajectory tracking performance, where robot end-effector follows the reference trajectory very well. Note the tracking errors could converge to zero in a short period of time as shown in Fig. 10. The trajectory of all joints angle is depicted in Fig.11. The applied virtual repulsive force from the obstacle on each joint is performed in Fig. 12. We can see that the applied forces maintain small value at first and then increase as the obstacle closing to the robot. Here, the effective of our proposed controller is verified.

\section{Conclusion}

This paper develops a novel algorithm for redundant robot control by using virtual fore field and null space projection. Two sub-controllers are designed to realized the control goal based on the decomposition of the system dynamics, namely the task space controller and the null space controller. Moreover, a minimum null space projection is employed to describe the null space motion, such that the virtual forces are project to the null space and will not affect the task space motion. An extra impedance controller is developed in the null space of the main task to guarantee compliant joint motion. A virtual force field is introduced and applied on the robot body to achieve the goal of the avoidance of the obstacle. Based on a 4 DOF planer robot, the simulation studies demonstrate that the proposed controller is not only to guarantee the task space control, but also able to avoid the obstacle by joint movements. 

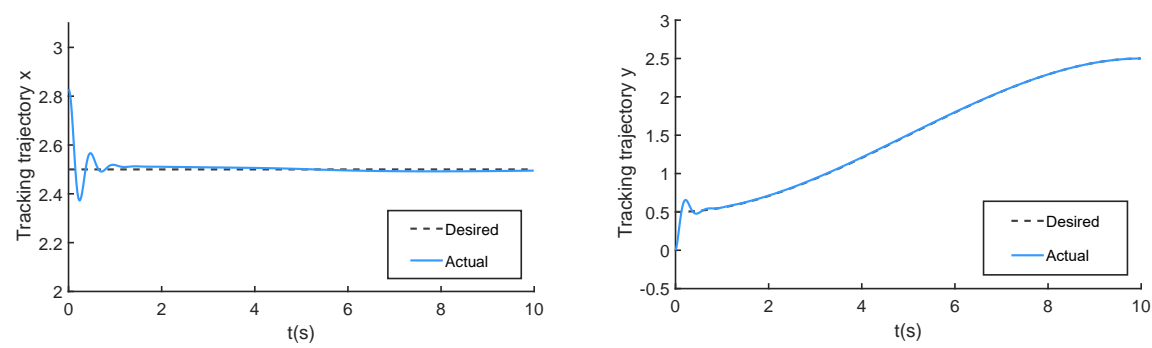

Fig. 8. Tracking trajectory of the proposed con- Fig. 9. Tracking trajectory of the proposed controller $x$ direction troller $y$ direction
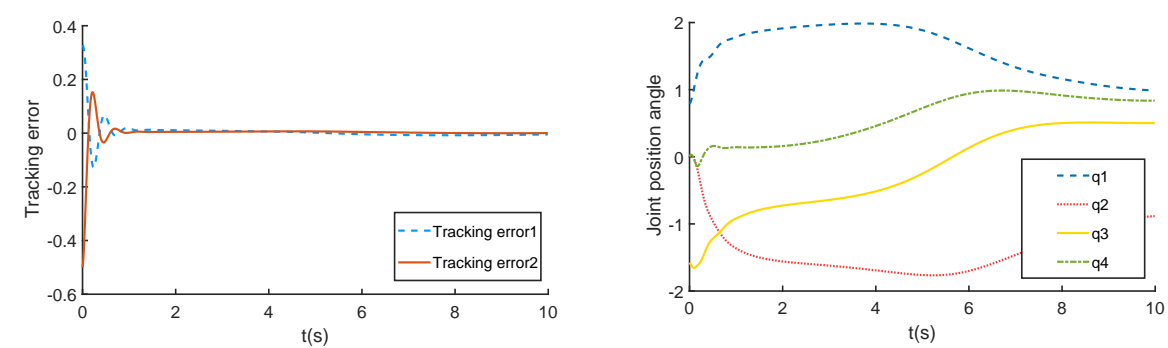

Fig. 10. Tracking error of the proposed con- Fig. 11. The joint angles of the robot during troller tracking

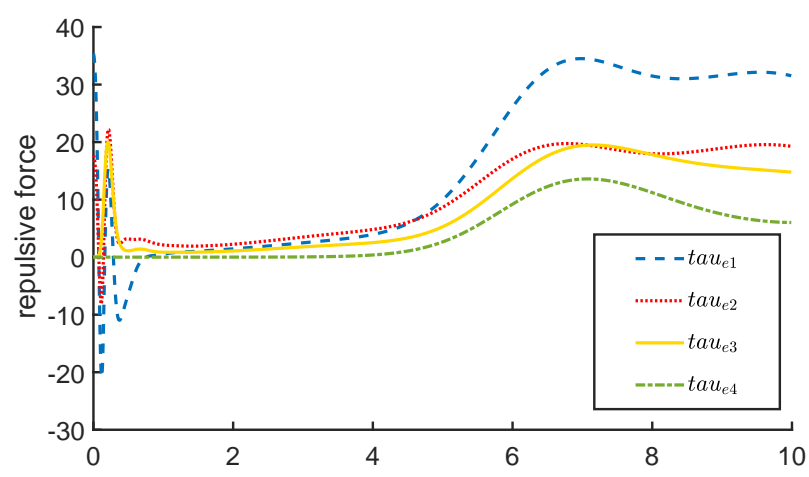

$t(s)$

Fig. 12. The applied repulsive force $\tau_{e}$ on each joint 


\section{Acknowledgement}

This work was partially supported by National Nature Science Foundation (NSFC) under Grants 61861136009 and 61811530281.

\section{References}

1. C. Yang, G. Ganesh, S. Haddadin, S. Parusel, A. Albu-Schaeffer, and E. Burdet, "Human-like adaptation of force and impedance in stable and unstable interactions," IEEE transactions on robotics, vol. 27, no. 5, pp. 918-930, 2011.

2. Y. Li, S. S. Ge, C. Yang, X. Li, and K. P. Tee, "Model-free impedance control for safe humanrobot interaction," in Robotics and Automation (ICRA), 2011 IEEE International Conference on. IEEE, 2011, pp. 6021-6026.

3. C. Yang, K. Huang, H. Cheng, Y. Li, and C.-Y. Su, "Haptic identification by elm-controlled uncertain manipulator," IEEE Transactions on Systems, Man, and Cybernetics: Systems, vol. 47, no. 8, pp. 2398-2409, 2017.

4. G. Tonietti, R. Schiavi, and A. Bicchi, "Design and control of a variable stiffness actuator for safe and fast physical human/robot interaction," in Robotics and Automation, 2005. ICRA 2005. Proceedings of the 2005 IEEE International Conference on. IEEE, 2005, pp. 526531.

5. K. Kong, J. Bae, and M. Tomizuka, "Control of rotary series elastic actuator for ideal force-mode actuation in human-robot interaction applications," IEEE/ASME transactions on mechatronics, vol. 14, no. 1, pp. 105-118, 2009.

6. A. Edsinger and C. C. Kemp, "Human-robot interaction for cooperative manipulation: Handing objects to one another," in Robot and Human interactive Communication, 2007. RO-MAN 2007. The 16th IEEE International Symposium on. IEEE, 2007, pp. 1167-1172.

7. K. L. Doty, C. Melchiorri, and C. Bonivento, "A theory of generalized inverses applied to robotics," The International Journal of Robotics Research, vol. 12, no. 1, pp. 1-19, 1993.

8. Y. Zhang, J. Wang, and Y. Xu, "A dual neural network for bi-criteria kinematic control of redundant manipulators," IEEE Transactions on Robotics and Automation, vol. 18, no. 6, pp. 923-931, 2002.

9. M. da Graça Marcos, J. T. Machado, and T.-P. Azevedo-Perdicoúlis, “Trajectory planning of redundant manipulators using genetic algorithms," Communications in nonlinear science and numerical simulation, vol. 14, no. 7, pp. 2858-2869, 2009.

10. P. Hsu, J. Mauser, and S. Sastry, "Dynamic control of redundant manipulators," Journal of Robotic Systems, vol. 6, no. 2, pp. 133-148, 1989.

11. B. Nemec, L. Žlajpah, and D. Omrčen, "Comparison of null-space and minimal null-space control algorithms," Robotica, vol. 25, no. 5, pp. 511-520, 2007.

12. Y. Nakamura, H. Hanafusa, and T. Yoshikawa, "Task-priority based redundancy control of robot manipulators," The International Journal of Robotics Research, vol. 6, no. 2, pp. 3-15, 1987.

13. B. Daachi, T. Madani, and A. Benallegue, "Adaptive neural controller for redundant robot manipulators and collision avoidance with mobile obstacles," Neurocomputing, vol. 79, pp. 50-60, 2012.

14. J. Baillieul, "Avoiding obstacles and resolving kinematic redundancy," in Proceedings. 1986 IEEE International Conference on Robotics and Automation, vol. 3. IEEE, 1986, pp. 16981704.

15. O. Khatib, "Real-time obstacle avoidance for manipulators and mobile robots," in $A u$ tonomous robot vehicles. Springer, 1986, pp. 396-404. 
12 Yiming Jiang, Chenguang Yang*, Zhaojie Ju, and Jinguo Liu

16. Y. K. Hwang and N. Ahuja, "A potential field approach to path planning," IEEE Transactions on Robotics and Automation, vol. 8, no. 1, pp. 23-32, 1992.

17. B. Nemec and L. Zlajpah, "Force control of redundant robots in unstructured environment," IEEE transactions on industrial electronics, vol. 49, no. 1, pp. 233-240, 2002.

18. L. E. Kavraki, P. Svestka, J. . Latombe, and M. H. Overmars, "Probabilistic roadmaps for path planning in high-dimensional configuration spaces," IEEE Transactions on Robotics and Automation, vol. 12, no. 4, pp. 566-580, Aug 1996.

19. S. M. LaValle, J. J. Kuffner, and Jr., "Rapidly-exploring random trees: Progress and prospects," 2000. 